\title{
Meta-analysis of the effect of perioperative intravenous lidocaine on return of gastrointestinal function after colorectal surgery
}

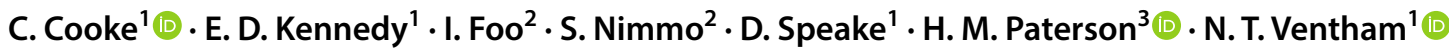

Received: 18 September 2018 / Accepted: 16 January 2019 / Published online: 5 February 2019

(c) The Author(s) 2019

\begin{abstract}
Background Return of normal gastrointestinal (GI) function is a critical determinant of recovery after colorectal surgery. The aim of this meta-analysis was to evaluate whether perioperative intravenous (IV) lidocaine benefits return of gastrointestinal function after colorectal resection.

Methods A comprehensive search of Ovid Medline, PubMed, Embase, Cochrane library, and clinicaltrials.org was performed on 1st July 2018. A manual search of reference lists was also performed. Inclusion criteria were as follows: randomized controlled trials (RCTs) of intravenous (IV) lidocaine administered perioperatively compared to placebo ( $0.9 \%$ saline infusion) as part of a multimodal perioperative analgesic regimen, human adults ( $>16$ years), and open or laparoscopic colorectal resectional surgery. Exclusion criteria: non-colorectal surgery, non-placebo comparator, children, non-general anaesthetic, and pharmacokinetic studies. The primary endpoint was time to first bowel movement. Secondary endpoints were time to first passage of flatus, time to toleration of diet, nausea and vomiting, ileus, pain scores, opioid analgesia consumption, and length of stay.

Results One hundred and ninety one studies were screened, with 9 RCTs meeting inclusion criteria (405 patients, four laparoscopic and five open surgery studies). IV lidocaine reduced time to first bowel movement compared to placebo [seven studies, 325 patients, mean weighted difference $-9.54 \mathrm{~h}, 95 \% \mathrm{CI} 18.72-0.36, p=0.04]$. Ileus, pain scores, and length of stay were reduced with IV lidocaine compared with placebo.

Conclusions Perioperative IV lidocaine may improve recovery of gastrointestinal function after colorectal surgery. Largescale effectiveness studies to measure effect size and evaluate optimum dose/duration are warranted.
\end{abstract}

Keywords Intravenous lidocaine $\cdot$ Colorectal surgery $\cdot$ Ileus $\cdot$ Laparoscopic

Electronic supplementary material The online version of this article (https://doi.org/10.1007/s10151-019-1927-1) contains supplementary material, which is available to authorized users.

H. M. Paterson

hugh.paterson@ed.ac.uk

1 Department of Colorectal Surgery, Western General Hospital, Edinburgh, UK

2 Department of Anaesthesia, Western General Hospital, Edinburgh, UK

3 Department of Colorectal surgery, Western General Hospital, University of Edinburgh Academic Coloproctology, Crewe Road South, Edinburgh EH4 2XU, UK

\section{Introduction}

Colorectal resection causes an unavoidable cessation of normal gastrointestinal (GI) function in every patient; hence, the return of GI function is a critical determinant of recovery $[1,2]$. Modern minimally invasive techniques and multimodal "enhanced recovery" programs have reduced the historically high prevalence of delayed return of GI function associated with open colorectal surgery [3,4]. Despite this, return of GI function after colorectal resection can lag behind other aspects of recovery such as mobilization and pain control [5]. A prolonged delay in return of GI function (commonly known as postoperative ileus) is characterized by inability to resume normal diet, vomiting, abdominal distension, and absolute constipation, requires active supportive management [intravenous (IV) fluids, anti-emetics, nasogastric intubation], and results in longer hospital stay 
with a substantially poorer patient experience. Recovery of GI function is important to patients and surgeons alike and was identified as a key research focus in a recent research prioritization exercise undertaken jointly between patients and the Association of Coloproctology of Great Britain and Ireland $[6,7]$.

Perioperative IV lidocaine has well-established antiinflammatory and opioid-sparing analgesic properties [8-10]. There are also data to suggest a beneficial effect on return of GI function following abdominal surgery. However, interpretation of the existing literature is challenging, as it includes a variety of operations, access techniques and perioperative management protocols [11, 12]. Furthermore, despite the existence of validated consensus-derived composite endpoint definitions of return of GI function (GI-2, GI-3) [2, 13, 14], many studies of perioperative IV lidocaine report a variety of sub-optimal univariate endpoints to measure GI recovery.

This study updates existing meta-analyses of perioperative IV lidocaine by inclusion of new data and seeks to limit study heterogeneity by focusing on return of GI function following colorectal surgery.

\section{Materials and methods}

\section{Literature search}

The study was placed prospectively on the International Prospective Register of Systematic Reviews (PROSPERO) register [CRD42016049847]. A comprehensive search of Ovid Medline, PubMed, Embase, Cochrane library, and clinicaltrials.org was completed on 5th September 2018. A manual search of reference lists was also performed. The following search strategy was used: (colorectal surgery OR colectomy OR colon OR colonic OR bowel) AND (intravenous lidocaine OR intravenous lignocaine OR lidocaine infusion OR lignocaine infusion OR IV lidocaine OR IV lignocaine OR I.V lidocaine).

\section{Inclusion criteria}

Randomized controlled trials (RCTs), human adults [>16 years], open or laparoscopic colorectal resectional surgery.

\section{Exclusion criteria}

Non-colorectal surgery, non-placebo comparator, children, non-general anaesthetic, and pharmacokinetic studies.

\section{Intervention and comparator}

Intravenous lidocaine administered perioperatively was compared to placebo ( $0.9 \%$ saline infusion) as part of a multimodal perioperative analgesic regimen.

\section{Data extraction}

Full-text randomized control trials meeting inclusion criteria were reviewed by two independent researchers (EK/CC). A proforma was used to extract relevant information: data presented as mean and standard deviation were extracted directly, whereas non-parametric results (median and interquartile range) were converted using previously described techniques. For skewed data, the median was used instead of the mean [15].

\section{Primary outcome}

Since none of the included studies reported the validated GI-2 or GI-3 definitions of GI function, the primary outcome was time (hours) to first bowel movement (various phrases "bowel function", "defecation", and "bowel motion" were used in the included studies and we have assumed them to mean the same thing, i.e., defecation).

\section{Secondary outcomes}

\section{Return of GI function}

- Time to first passage of flatus (hours).

- Time to toleration of diet (hours).

- Incidence of postoperative ileus.

- Incidence of nausea and vomiting.

Pain

- Numerical pain score at rest at $24 \mathrm{~h}$ (score $0-10,0=$ no pain, 10 worst imaginable pain, alternative methods converted to $0-10$ range).

- Numerical pain score on movement at $24 \mathrm{~h}(0-10$ as above).

- Opioid consumption over first $24 \mathrm{~h}$ after surgery (milligrams and morphine equivalent doses).

- Total opioid consumption (milligrams).

Other

- Length of stay (hours). 


\section{Subgroup analyses}

A predefined subgroup analysis was performed for open and laparoscopic surgery.

\section{Bias and quality assessment}

Overall quality and potential bias were assessed using a previously described 15 -point scale adapted from criteria described by Chalmers and Jadad, with a threshold score of $\geq 12$ for high quality (Table 1) [16, 17]. A sensitivity analysis was conducted for the primary endpoint by excluding each study.

\section{Statistical analysis}

Data were analyzed using the mean weighted difference (WMD) and pooled odds ratios for continuous variables and dichotomous data, respectively. A random effects model was selected on the basis of radial plots of the primary outcome. Statistical significance was set at $p<0.05$. Heterogeneity was classified as low $(<33 \%)$, medium (33-66), or high ( $>66 \%$ ) using I2 estimated using the restricted maximum likelihood estimator function. Data were analyzed using the metafor package in $\mathrm{R}$ (version 3.4.2, $\mathrm{R}$ statistical programming, Vienna) [26].

\section{Results}

Nine RCTs were identified by the literature search strategy detailed in the Preferred Reporting Items for Systematic Reviews and Meta-analyses (PRISMA) flow diagram in Fig. 1, with a total of 405 patients. All results and figures are presented in supplementary data.

\section{Primary outcome: time to first bowel movement}

IV lidocaine was associated with a significantly reduced time to first bowel movement (Fig. 2) in pooled analysis compared with placebo (seven studies, 325 patients, WMD $-9.54 \mathrm{~h}, 95 \%$ CI 18.72 to $-0.36, p=0.04)$. In subgroup analyses, IV lidocaine reduced time to first bowel movement in both open (three studies, 139 patients; WMD $-14.87 \mathrm{~h}$, 95\% CI 25.92 to $-3.81, p=0.008$ ) and laparoscopic surgery (four studies, 186 patients; WMD $-12.1 \mathrm{~h}, 95 \% \mathrm{CI} 23.6$ to $-0.59, p=0.04)$.

\section{Secondary outcomes}

\section{Time to first passage of flatus}

Intravenous lidocaine did not significantly improve time to first passage of flatus in pooled analysis ( 8 studies, 345 patients; WMD $-3.42 \mathrm{~h}, 95 \% \mathrm{CI} 10.41-3.58, p=0.339$ ). There was a significant decrease in the time to first passage of flatus in the open subgroup (five studies, 229 patients; WMD $-7.07 \mathrm{~h}, 95 \%$ CI 13.58 to $-0.57, p=0.033$ ) but not the laparoscopic subgroup (three studies, 126 patients; WMD -4.58 h, $95 \%$ CI -18.22 to $9.07, p=0.511$ ).

\section{Time to resumption of diet}

Only three studies reported this endpoint. IV lidocaine did not significantly hasten the time to toleration of diet in pooled analysis (three studies, 188 patients; WMD$10.93 \mathrm{~h}, 95 \% \mathrm{CI}-23.03$ to $1.17, p=0.077)$. IV lidocaine was associated with a shorter time to toleration of diet compared with placebo only in the laparoscopic subgroup (two studies, 128 patients; WMD $-5.97 \mathrm{~h}, 95 \% \mathrm{CI}-6.88$ to -5.09 , $p<0.001)$. However, this was heavily weighted by a single study. Furthermore, resumption of diet is a less objective measurement than return of bowel function, as it greatly varies by individual practice.

\section{Nausea and vomiting}

There was no significant difference in nausea and vomiting events when comparing IV lidocaine with placebo in pooled analysis (five studies, 271 patients, OR $0.54,95 \% \mathrm{CI}$ $0.21-1.41, p=0.150)$. There was no significant difference in the laparoscopic and open subgroups.

\section{Incidence of postoperative ileus}

In pooled analysis, there was a significant reduction in the incidence of postoperative ileus in the IV lidocaine group (five studies, 256 patients, OR $0.32,95 \%$ CI $0.15-0.71$, $p=0.02$ ). No differences in the incidence of postoperative ileus were seen in subgroup analyses.

\section{Pain score at rest at $24 \mathrm{~h}$}

Intravenous lidocaine was associated with lower pain scores at rest at $24 \mathrm{~h}$ compared with placebo (seven studies, 280 patients, WMD $-0.72,95 \% \mathrm{CI}-1.31$ to $-0.13, p=0.020$ ). This benefit was seen in the open subgroup (four studies, 159 patients, WMD $-0.36,95 \% \mathrm{CI}-0.66$ to $-0.06, p=0.02$ ). There was no significant difference in the laparoscopic subgroup. 


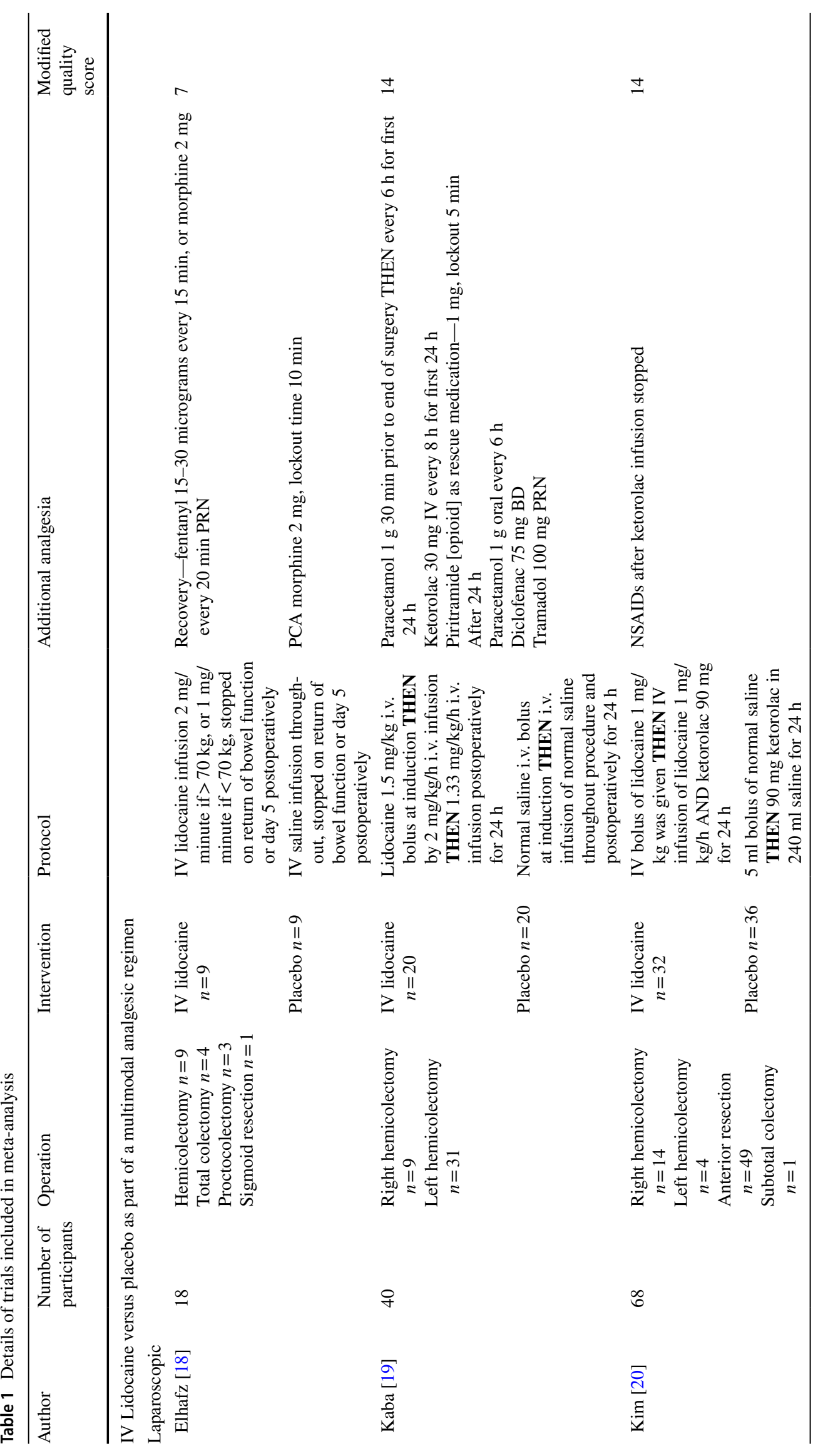




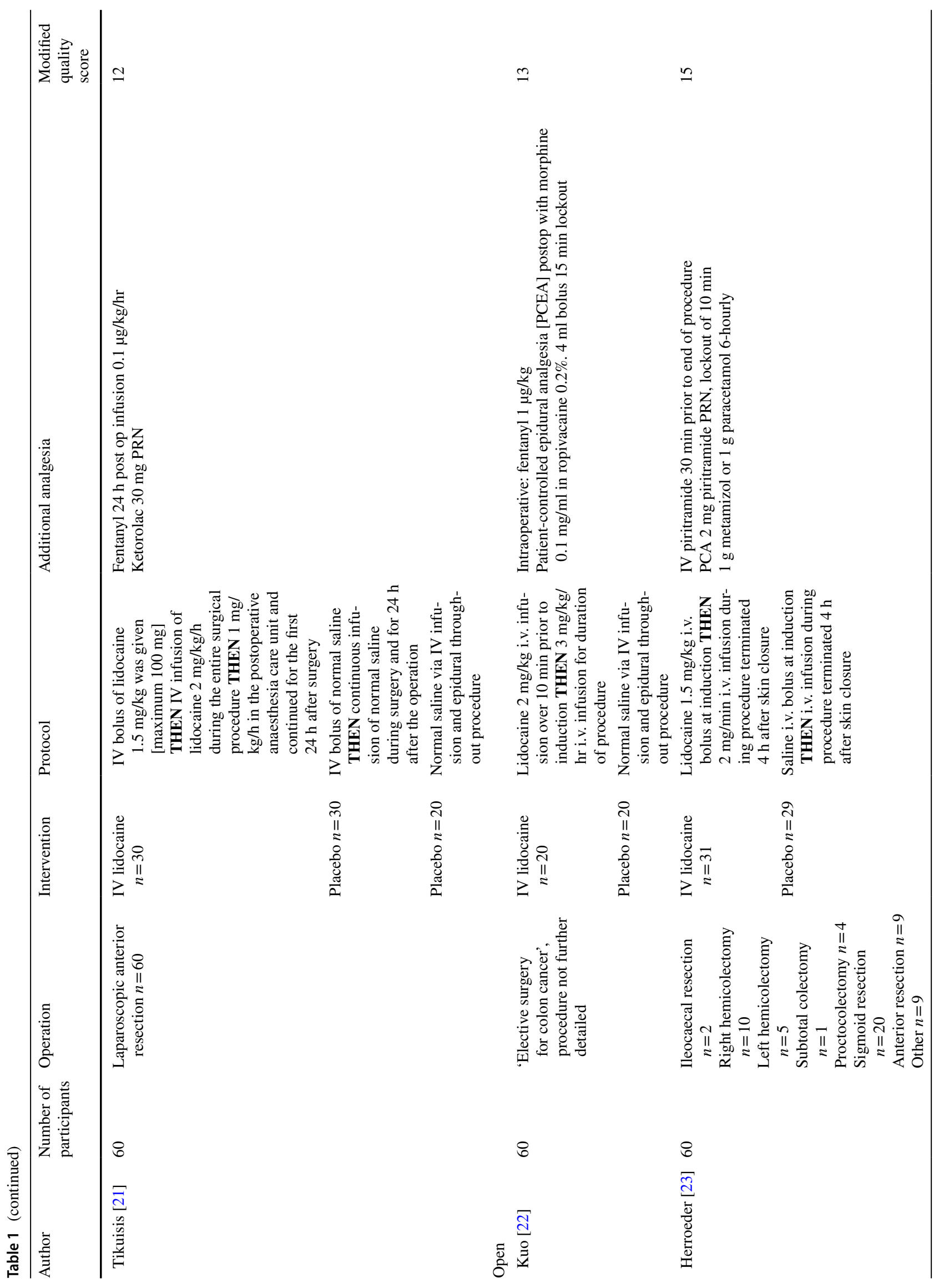




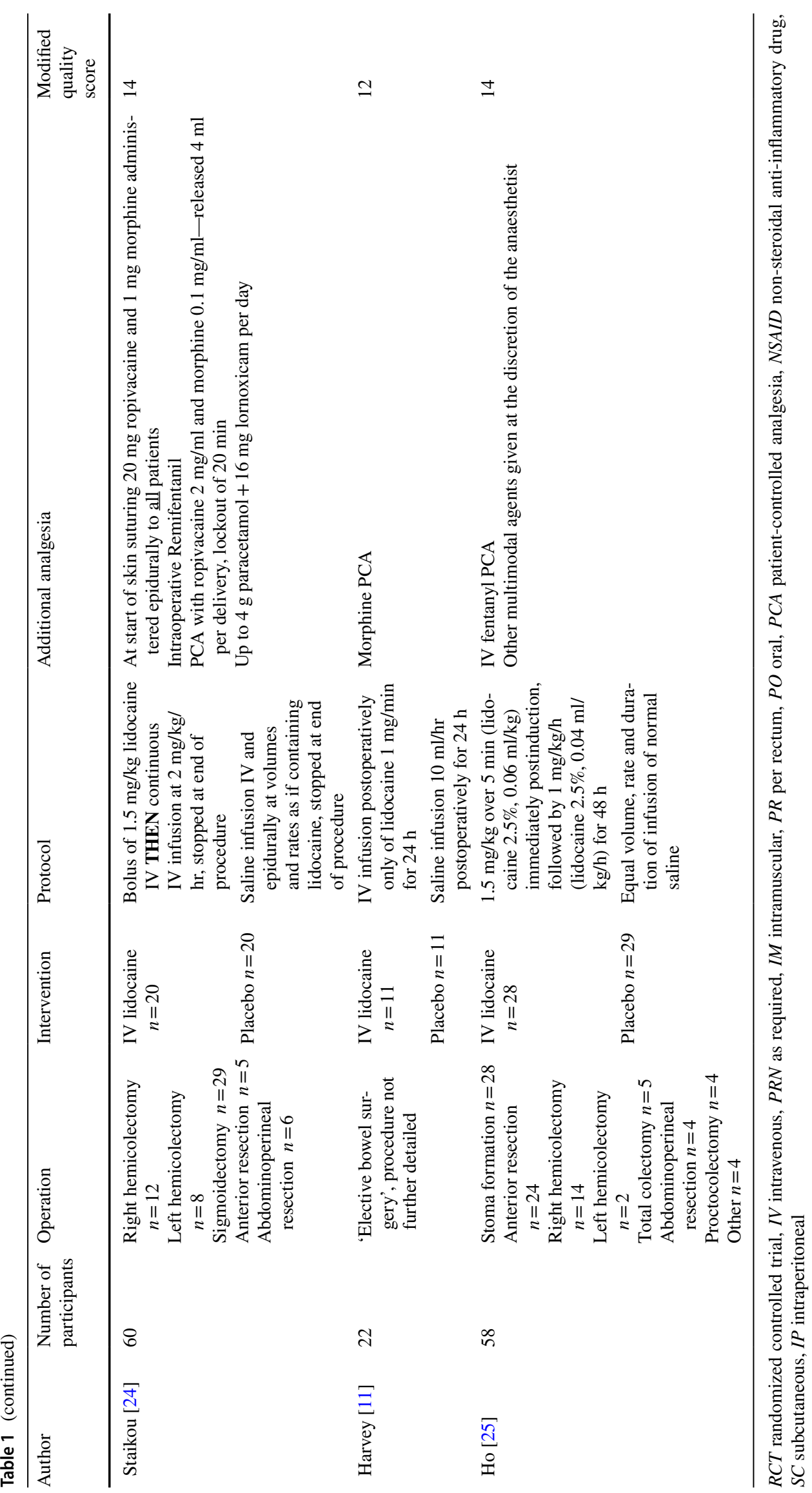


Fig. 1 PRISMA flow diagram of search strategy and included studies
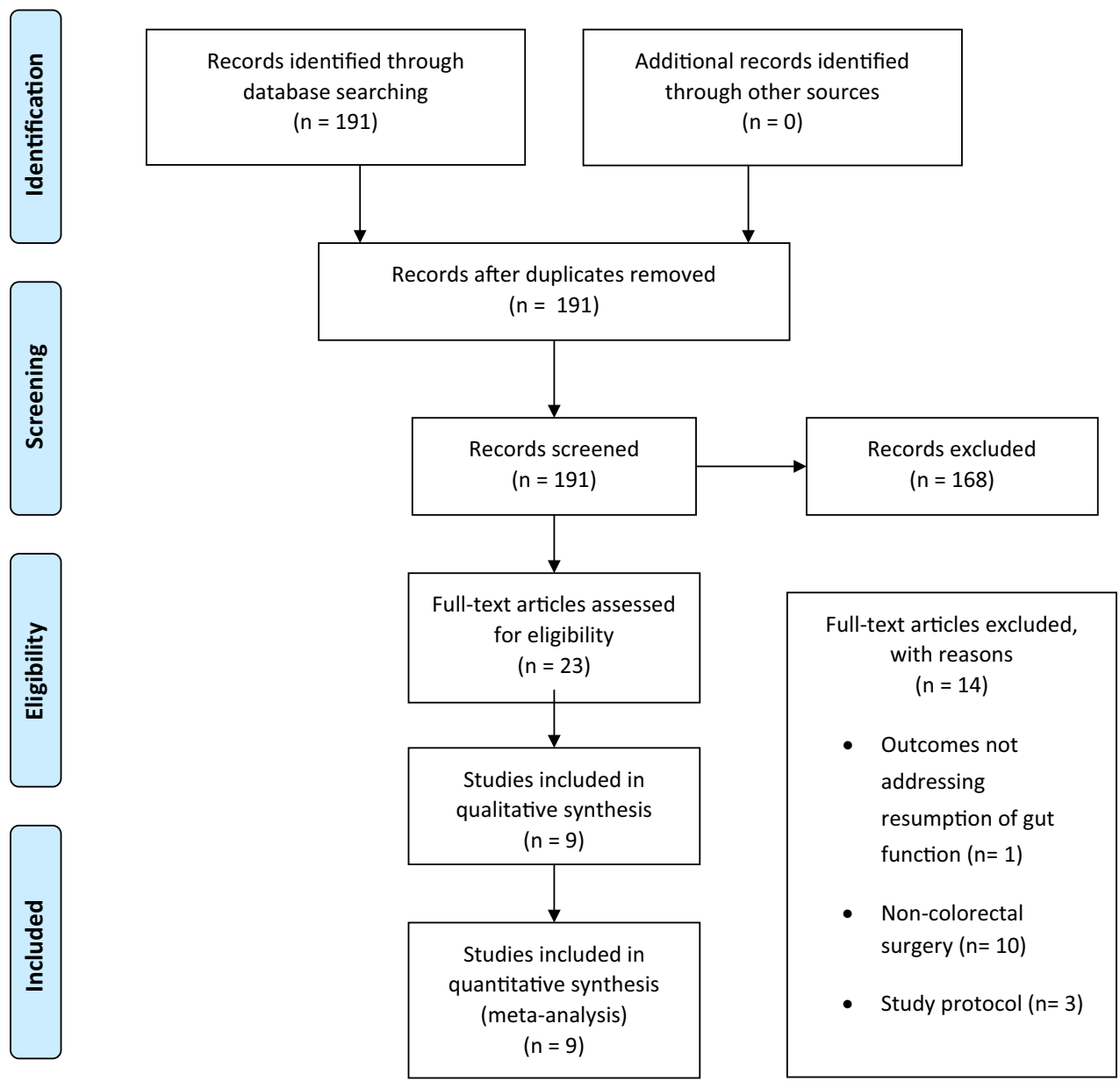

\section{Pain score on movement at $24 \mathrm{~h}$}

Intravenous lidocaine was associated with lower pain scores on movement at $24 \mathrm{~h}$ compared with placebo (four studies, 133 patients, WMD $-1.02, \mathrm{CI}-1.89$ to $-0.14, p=0.020$ ). This effect was seen in the laparoscopic (two studies, WMD $-1.70,95 \%$ CI -2.18 to $-1.22, p<0.0001)$ but not the open subgroup (two studies, 80 patients, WMD $-0.38,95 \% \mathrm{CI}$ -1.06 to $0.31, p=0.28)$.

\section{Opioid consumption during first $24 \mathrm{~h}$ after operation}

There was no difference in opioid consumption in the first $24 \mathrm{~h}$ after operation in pooled or subgroup analyses (pooled analysis five studies, 205 patients; WMD $-4.24 \mathrm{mg}$, 95\%CI -9.86 to $1.38, p=0.14)$.

\section{Total opioid consumption}

There was no significant difference in total opioid consumption in pooled or subgroup analyses (pooled analysis seven studies, 305 patients; WMD $-5.82 \mathrm{mg}, 95 \% \mathrm{CI}-22.32$ to $10.67, p=0.49$ ).

\section{Length of stay}

Intravenous lidocaine was associated with shorter length of stay in pooled analysis (seven studies, 347 patients; WMD $-17.84 \mathrm{~h}, 95 \% \mathrm{CI}-32.95$ to $-2.74 \mathrm{~h}, p=0.020$ ). This was the case in both laparoscopic (three studies, 168 patients; WMD $-23.04 \mathrm{~h}, 95 \% \mathrm{CI}-32.52$ to $-13.56 \mathrm{~h}, p<0.0001$ ) and open subgroups (four studies, 179 patients; WMD $-19.62 \mathrm{~h}, 95 \% \mathrm{CI}-36.66$ to $-2.59 \mathrm{~h}, p=0.020$ ).

Forest plots for secondary outcomes are shown in Supplementary data 1 .

\section{Discussion}

Previous meta-analyses of perioperative IV lidocaine have included a diverse range of operative procedures and focused on opioid analgesic consumption and pain scores [27, 28]. This meta-analysis examined the effect of IV lidocaine on return of GI function after major colorectal surgery, a critical determinant of recovery and discharge from hospital for this patient group. Time to first bowel movement was reduced by approximately $15 \mathrm{~h}$ in open and $12 \mathrm{~h}$ in laparoscopic surgery. 


\section{Time until resumption of Bowels}

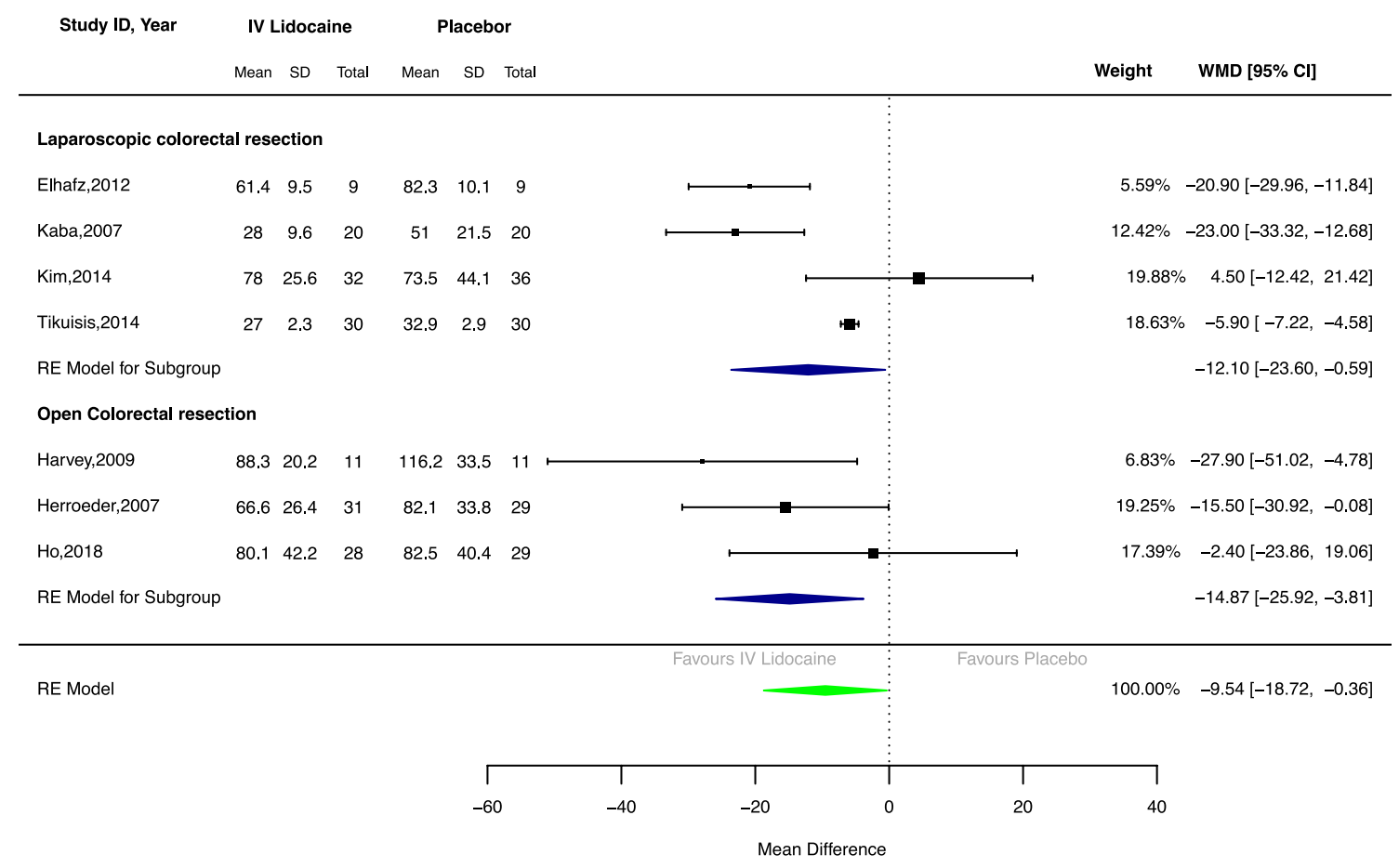

Fig. 2 Forest plot of time from operation to first bowel movement

Consistent with this finding was a substantially reduced risk of postoperative ileus (OR 0.32), reduced early pain scores, and reduced length of hospital stay of approximately $18 \mathrm{~h}$ ( $95 \% \mathrm{CI}-2.74$ to $-32.95 \mathrm{~h}$ ). If these findings were replicated in routine practice, perioperative IV lidocaine could hasten recovery, reduce postoperative ileus, and reduce length of stay for a significant proportion of patients. Given that colectomy is a common operation undertaken in every acute hospital in the western world, considerable cost savings could be achieved in reduced bed occupancy from this straightforward and inexpensive intervention. Although this analysis did not specifically study the safety of IV lidocaine, it is a familiar drug and the previous reviews suggest a low incidence of IV lidocaine-associated toxicity [29].

The mechanism of action of IV lidocaine in this setting remains uncertain. Pain scores were lower with IV lidocaine, but opioid consumption was not significantly different, suggesting that the faster return of gut function was not solely due to opiate sparing [30, 31]. IV lidocaine has a variety of analgesic and anti-inflammatory effects mediated through sodium channel receptors (recently summarized in detail [32]) and is known to reduce postoperative serum cytokine levels, suggesting that it acts centrally and peripherally to blunt the pro-inflammatory response to surgery $[18,33]$. Postoperative ileus is multifactorial, and IV lidocaine probably acts via more than one mechanism.
Our study aimed to highlight potential benefits of perioperative IV lidocaine to colorectal surgeons, but has several limitations and its results need to be interpreted carefully. No study reported the consensus-derived, validated GI-2 or GI-3 composite endpoints of GI function and not all univariate endpoints were reported by all studies (for example, time to resumption of diet, integral to the GI2/GI-3 endpoint, was reported by only three studies). Although dose was consistent between studies (1-2 mg/ $\mathrm{kg} / \mathrm{h}$ ), duration of infusion was not: most studies used $24 \mathrm{~h}$, but ranged from operation only [22, 24] to 5 days (Elhafz et al. [18]). The latter study is, therefore, a methodological outlier, elimination of which in sensitivity analysis (Supplementary data 2) leads to a loss of statistical significance for the laparoscopic subgroup for the primary endpoint. This sensitivity analysis shows that our results are susceptible to removal of individual studies, reflecting study heterogeneity and the small total sample size, and is another reason for cautious interpretation.

Currently, the 'correct' duration of infusion is unknown. The intraoperative period is probably the most important; thereafter, continuation of the infusion depends on availability of cardiac monitoring beyond the theatre suite, which may be dictated by local resources. Plasma accumulation, and hence risk of toxicity, is unlikely with less than $24 \mathrm{~h}$ continuous infusion [32]. The authors' local practice is a 12-h infusion, and the UK ALLEGRO trial 
of perioperative IV lidocaine will compare outcomes from 6-h and 12-h infusion [34].

Finally, few studies reported a perioperative protocol consistent with modern enhanced recovery principles. Notably, those that did had short lengths of stay (median 3-4 days) and showed a clear benefit from IV lidocaine $[19,21]$. In contrast, where enhanced recovery protocols were not used/reported and length of stay was longer (median 8-9 days), no benefit was shown [20, 25]. This suggests that IV lidocaine exerted the greatest benefit on early recovery and was most effective within a modern patient care protocol; conversely, where length of stay was long (outdated care pathways, complex case mix, or high complication rates), a benefit was more difficult to detect.

\section{Conclusions}

Although this analysis reduces heterogeneity by including colorectal surgery only, most studies were small, set in contrasting perioperative care protocols and reported suboptimal endpoints to assess postoperative GI function. Nevertheless, an intriguing signal of benefit from IV lidocaine was seen consistently across the reported outcomes, suggesting that perioperative IV lidocaine could have a clinically meaningful effect on return of GI function, and hence, length of stay after colorectal surgery. IV lidocaine is inexpensive, straightforward to administer within existing evidence-based perioperative care protocols, and appears safe. Large-scale pragmatic effectiveness trials embedded within modern perioperative protocols are warranted to confirm or refute these findings and optimize dose and duration of infusion.

Author contributions CC: conception, literature searches, data synthesis, data analysis, and write up. EDK: literature searches, data synthesis, and approval of final manuscript. SN: conception, editing, and approval of final manuscript. IF: conception, editing, and approval of final manuscript. DS: conception, editing, and approval of final manuscript. HMP: conception, editing, and approval of final manuscript. NTV: conception, data analysis, write-up, editing, and approval of final manuscript.

Funding No funding obtained for this study.

\section{Compliance with ethical standards}

Conflict of interest HMP, DS, SN, and IF are lead investigators in a UK National Institute for Health Research Health Technology Assessment (NIHR-HTA)-funded UK multicenter randomized controlled trial of perioperative intravenous lidocaine in colorectal surgery (ALLEGRO: a placebo-controlled rAndomized trial of intravenous Lidocaine in acceLErating Gastrointestinal Recovery after cOlorectal surgery; EudraCT No. 2017-003835-12), which opened in August 2018 and will randomize 562 patients undergoing laparoscopic colorectal resection, measuring GI-3 return of GI function as the primary endpoint. Further details are available at: https://w3.abdn.ac.uk/hsru/ALLEGRO/Public/ Public/index.cshtml. None of the authors have any commercial interest in perioperative IV lidocaine.

Declaration of originality This manuscript has not been submitted elsewhere. An abstract based on this data was presented as a poster at the 2017 annual meeting of the Association of ColoProctologists of Great Britain and Ireland in Bournemouth, UK.

Ethical approval This article does not contain any studies with human participants or animals performed by the authors.

Informed consent For this type of study informed consent is not required.

Open Access This article is distributed under the terms of the Creative Commons Attribution 4.0 International License (http://creativeco mmons.org/licenses/by/4.0/), which permits unrestricted use, distribution, and reproduction in any medium, provided you give appropriate credit to the original author(s) and the source, provide a link to the Creative Commons license, and indicate if changes were made.

\section{References}

1. Delaney CP, Marcello PW, Sonoda T, Wise P, Bauer J, Techner L (2010) Gastrointestinal recovery after laparoscopic colectomy: results of a prospective, observational, multicenter study. Surg Endosc 24:653-661

2. Delaney CP, Kehlet H, Senagore A, Bauer A, Beart R, Billingham R (2006) Postoperative ileus: profiles, risk factors, and definitions: a framework for optimizing surgical outcomes in patients undergoing major abdominal colorectal surgery. Clinical consensus update in general surgery. Pharmacolecture LLC, Roswell (GA)

3. Khoury W, Dakwar A, Sivkovits K, Mahajna A (2014) Fast-track rehabilitation accelerates recovery after laparoscopic colorectal surgery. J Soc Laparoendosc Surg 18(4): e2014.00076

4. Artinyan A, Nunoo-Mensah JW, Balasubramaniam S, Gauderman J, Essani R, Gonzalez-Ruiz C et al (2008) Prolonged postoperative ileus-definition, risk factors, and predictors after surgery. World J Surg 32:1495-1500

5. Creamer F, Balfour A, Nimmo S, Foo I, Norrie JD, Williams LJ et al (2017) Randomized open-label phase II study comparing oxycodone-naloxone with oxycodone in early return of gastrointestinal function after laparoscopic colorectal surgery. Br J Surg 104:42-51

6. McNair AG, Heywood N, Tiernan J, Verjee A, Bach SP, Fearnhead NS et al (2017) A national patient and public colorectal research agenda: integration of consumer perspectives in bowel disease through early consultation. Colorectal Dis 19:O75-O85

7. Tiernan J, Cook A, Geh I, George B, Magill L, Northover J et al (2014) Use of a modified Delphi approach to develop research priorities for the association of coloproctology of Great Britain and Ireland. Colorectal Dis 16:965-970

8. Marret E, Rolin M, Beaussier M, Bonnet F (2008) Meta-analysis of intravenous lidocaine and postoperative recovery after abdominal surgery. Br J Surg 95:1331-1338

9. Vigneault L, Turgeon AF, Cote D, Lauzier F, Zarychanski R, Moore L et al (2011) Perioperative intravenous lidocaine infusion for postoperative pain control: a meta-analysis of randomized controlled trials. Can J Anaesth 58:22-37 
10. Hollmann MW, Durieux ME (2000) Local anesthetics and the inflammatory response: a new therapeutic indication? Anesthesiology 93:858-875

11. Harvey KP, Adair JD, Isho M, Robinson R (2009) Can intravenous lidocaine decrease postsurgical ileus and shorten hospital stay in elective bowel surgery? A pilot study and literature review. Am J Surg 198:231-236

12. Ventham NT, Kennedy ED, Brady RR, Paterson HM, Speake D, Foo I et al (2015) Efficacy of intravenous lidocaine for postoperative analgesia following laparoscopic surgery: a meta-analysis. World J Surg 39:2220-2234

13. Delaney CP, Weese JL, Hyman NH, Bauer J, Techner L, Gabriel $\mathrm{K}$ et al (2005) Phase III trial of alvimopan, a novel, peripherally acting, mu opioid antagonist, for postoperative ileus after major abdominal surgery. Dis Colon Rectum 48:1114-1125; (discussion 25-6; author reply 27-9)

14. Ludwig K, Enker WE, Delaney CP, Wolff BG, Du W, Fort JG et al (2008) Gastrointestinal tract recovery in patients undergoing bowel resection: results of a randomized trial of alvimopan and placebo with a standardized accelerated postoperative care pathway. Arch Surg 143:1098-1105

15. Hozo SP, Djulbegovic B, Hozo I (2005) Estimating the mean and variance from the median, range, and the size of a sample. BMC Med Res Methodol 5:13

16. Chalmers TC, Smith H Jr, Blackburn B, Silverman B, Schroeder B, Reitman D et al (1981) A method for assessing the quality of a randomized control trial. Control Clin Trials 2:31-49

17. Jadad AR, Moore RA, Carroll D, Jenkinson C, Reynolds DJ, Gavaghan DJ et al (1996) Assessing the quality of reports of randomized clinical trials: is blinding necessary? Control Clin Trials 17:1-12

18. Elhafz AA, Elgebaly AS, Bassuoni AS, El Dabaa AA (2012) Is lidocaine patch as effective as intravenous lidocaine in pain and illus reduction after laparoscopic colorectal surgery? A randomized clinical trial. Anesth Essays Res 6:140-146

19. Kaba A, Laurent S, Detroz B, Sessler D, Durieux M, Lamy M et al (2007) Intravenous lidocaine infusion facilitates acute rehabilitation after laparoscopic colectomy. Anesthesiology 106:11-18

20. Kim HO, Lee SR, Choi WJ, Kim H (2014) Early oral feeding following laparoscopic colorectal cancer surgery. ANZ J Surg 84:539-544

21. Tikuisis R, Miliauskas P, Samalavicius N (2014) Intravenous lidocaine for post-operative pain relief after hand-assisted laparoscopic colon surgery: a randomized, placebo-controlled clinical trial. Tech Coloproctol 18:373-380

22. Kuo CP, Jao SW, Chen KM, Wong CS, Yeh CC, Sheen MJ et al (2006) Comparison of the effects of thoracic epidural analgesia and i.v. infusion with lidocaine on cytokine response, postoperative pain and bowel function in patients undergoing colonic surgery. Br J Anaesth 97:640-646
23. Herroeder S, Pecher S, Schonherr ME, Kaulitz G, Hahnenkamp $\mathrm{K}$, Friess H et al (2007) Systemic lidocaine shortens length of hospital stay after colorectal surgery: a double-blinded, randomized, placebo-controlled trial. Ann Surg 246:192-200

24. Staikou C, Avramidou A, Ayiomamitis GD, Vrakas S, Argyra E (2014) Effects of intravenous versus epidural lidocaine infusion on pain intensity and bowel function after major large bowel surgery: a double-blind randomized controlled trial. J Gastrointest Surg 18:2155-2162

25. Ho MLJ, Kerr SJ, Stevens J (2018) Intravenous lidocaine infusions for 48 hours in open colorectal surgery: a prospective, randomized, double-blinded, placebo-controlled trial. Korean J Anesthesiol 71:57-65

26. Viechtbauer W (2010) Conducting meta-analyses in R with the metafor package. J Stat Soft 36(3):1-48. https://www.jstatsoft.org/ v036/i03

27. Sun Y, Li T, Wang N, Yun Y, Gan T (2012) Perioperative systemic lidocaine for postoperative analgesia and recovery after abdominal surgery: a meta-analysis of randomized controlled trials. Dis Colon Rectum 55:1183-1194

28. Tremont-Lukats IW, Challapalli V, McNicol ED, Lau J, Carr DB (2005) Systemic administration of local anesthetics to relieve neuropathic pain: a systematic review and meta-analysis. Anesth Analg 101:1738-1749

29. Weibel S, Jelting Y, Pace NL, Helf A, Eberhart LH, Hahnenkamp $\mathrm{K}$ et al (2018) Continuous intravenous perioperative lidocaine infusion for postoperative pain and recovery in adults. Cochrane Database Syst Rev 6:CD009642

30. Kurz A, Sessler DI (2003) Opioid-induced bowel dysfunction: pathophysiology and potential new therapies. Drugs 63:649-671

31. Bauer AJ, Boeckxstaens GE (2004) Mechanisms of postoperative ileus. Neurogastroenterol Motil 16(Suppl 2):54-60

32. Beaussier M, Delbos A, Maurice-Szamburski A, Ecoffey C, Mercadal L (2018) Perioperative use of intravenous lidocaine. Drugs 78:1229-1246

33. Song X, Sun Y, Zhang X, Li T, Yang B (2017) Effect of perioperative intravenous lidocaine infusion on postoperative recovery following laparoscopic cholecystectomy-a randomized controlled trial. Int J Surg 45:8-13

34. ALLEGRO: A placebo controlled randomised trial of intravenous lidocaine in accelerating gastrointestinal recovery after colorectal surgery. https://w3.abdn.ac.uk/hsru/ALLEGRO/Public/Publi c/index.cshtml

Publisher's Note Springer Nature remains neutral with regard to jurisdictional claims in published maps and institutional affiliations. 